\title{
Erratum to: Musculoskeletal manifestations of primary hyperparathyroidism
}

\author{
Ramesh Pappu $^{1}$ - Serge A. Jabbour ${ }^{2}$ - Anthony M. Reginato ${ }^{3}$ - Antonio J. Reginato ${ }^{4}$
}

Published online: 14 January 2017

(C) International League of Associations for Rheumatology (ILAR) 2017

Erratum to: Clin Rheumatol (2016) 35:3081-3087

DOI 10.1007/s10067-016-3450-3

The author name Anthony M. Reginato and his affiliation were incorrectly listed in the original publication. The author name and affiliation has been corrected here. The remainder of the original article content remains unchanged.

The online version of the original article can be found at http://dx.doi. org/10.1007/s10067-016-3450-3.

Ramesh Pappu

pappur@aol.com

1 College of Medicine, Drexel University, 414 Downs Drive, Cherry Hill, NJ 08003, USA

2 Thomas Jefferson University, 233 South Tenth Street, Philadelphia, PA 19107, USA

3 Division of Rheumatology, Providence VAMC, Warren Alpert School of Medicine, Brown University, 830 Chalkstone Ave, Providence, RI 02908, USA

4 Head Rheumatology Section, Cooper Hospital/University Medical Center, Robert Wood Johnson Medical School, Rutgers University, Camden, NJ 08103, USA 